\title{
Positioning uncertainty of the Tricept type parallel kinematic structure
}

\author{
Eva Kureková ${ }^{1}$, Milada Omachelová ${ }^{1}$, Martin Halaj ${ }^{2}$, Ilja Martišovitš ${ }^{3}$ \\ ${ }^{1}$ Faculty of Mechanical Engineering, Slovak University of Technology, Bratislava, Slovakia \\ ${ }^{2}$ Bratislava, Slovakia \\ ${ }^{3}$ Microstep spol. s.r.o., Bratislava, Slovakia
}

\begin{abstract}
The paper discusses theoretical aspects that arose during the determination of the theoretical positioning accuracy of parallel kinematic structures (PKS) with special regard to the Tricept type PKS. Apart from the conventional serial structures that employ translational or rotational movement (or a combination) of individual driving axes. Parallel structures comprise a set of telescopic driving rods that are joined together via a solid platform. Due to this fact, the mathematical model describing the relationship between the driving actions of the telescopic rods and the resulting coordinates of the desired effector's endpoint is rather complex. To determine the theoretically achievable positioning accuracy of the endpoint, authors investigated the theoretical influence of geometrical imperfections of the machine design and employed the law of uncertainties propagation. The aim was to investigate theoretically the achievable positioning accuracy of the machine, prior to the final design solutions, thus helping the designer to optimize the machine's design.
\end{abstract}

\section{Section: RESEARCH PAPER}

Keywords: parallel kinematic structures; Tricept; positioning accuracy; coordinates uncertainty

Citation: Eva Kureková, Milada Omachelová, Martin Halaj, Ilja Martišovitš, Positioning uncertainty of the Tricept type parallel kinematic structure, Acta IMEKO, vol. 4, no. 4, article 4, December 2015, identifier: IMEKO-ACTA-04 (2015)-04-04

Section Editor: Franco Pavese, Torino, Italy

Received April 2, 2015; In final form May 19, 2015; Published December 2015

Copyright: () 2015 IMEKO. This is an open-access article distributed under the terms of the Creative Commons Attribution 3.0 License, which permits unrestricted use, distribution, and reproduction in any medium, provided the original author and source are credited

Corresponding author: Eva Kureková, e-mail: eva.kurekova@stuba.sk

\section{INTRODUCTION}

Parallel kinematic structures (PKS) represent a nonconventional way for the arrangement of movement elements, compared to the widely used serial kinematic structures. They employ parallel arranged movement elements (telescopic rods, arms) that have one end located at a base frame and the second end connected to a movable platform. The Tricept belongs among the most known PKS [1]-[4]. It is a fixed platform connected to a movable platform via three driving telescopic rods and a non-driven central rod (Figure 1). The central rod is connected to a movable platform by a solid linkage, while it can move axially against the fixed platform (rotation of the central rod is prevented). The effector is usually connected to a movable platform, carrying the tools or technological heads. A servomotor located at the end of each telescopic rod enables extension of the rod by a ball screw and nuts. The skeleton together with a primary platform create a single kinematic element [1]-[9].

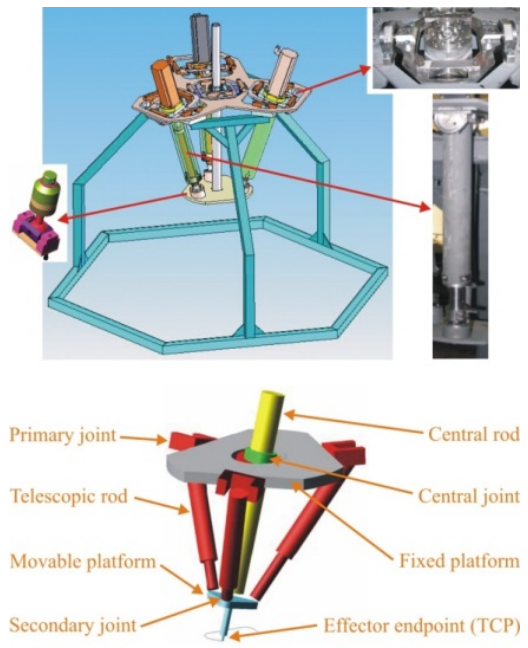

Figure 1. Parallel kinematic structure of the Tricept type. 


\section{REACHING THE DESIRED POSITION OF THE END EFFECTOR}

To control the position of the Tricept end effector, one must necessarily know the relation between the extensions of the individual telescopic rods and the position of the effector's endpoint. A mathematical model of this relation is described in [7] and [8]. The positioning of the effector's endpoint is carried out by rotating the movable platform about the axes $x$ and $y$ together with its shifting along the $z$ axis. As the movement of the end point generates an irregular workspace, possible singularities and collision points must be investigated [10].

Let $Q_{q}$ be the reference point, tightly connected with the movable platform (i.e. static against point $P$ ), whose position will be investigated. To reach the defined position of point $Q$ in the workspace, the movable platform has to turn around the $x$ and $y$ axes and shift along the $z$ axis.

We obtain the cartesian positions $Q=\left[Q_{x}, Q_{1}, Q_{3}\right]$ of point $Q_{q}$ (relative to the "static" coordinate system bound with the static platform (relative to point P) by applying the next three transformations in the following order (on $\left.\left[q_{x}, q_{y}, q_{2}\right]\right)$ :

1. translation along the $z$ axis (so coordinates of point $Q_{q}$ relative to "static" coordinate system are changed to $\left.\left[\boldsymbol{q}_{x}, \boldsymbol{q}_{\boldsymbol{y}}, \boldsymbol{q}_{z}+z_{\boldsymbol{e}}\right]\right)$

2. rotation about the $x$ axis by the angle $\alpha$ (represented by orthogonal matrix $\left.\boldsymbol{O}_{x}(\alpha)\right)$;

3. rotation about the $y$ axis by the angle $\beta$ (represented by orthogonal matrix $\left.\boldsymbol{O}_{y}(\beta)\right)$;

where (in general) angles $\alpha, \beta$ and shift $z$ are non-zero. After applying these three transformations we obtain the Cartesian coordinates $Q=\left[Q_{x}, Q_{y}, Q_{z}\right]$ of point $Q_{q}$ in the "static" coordinate system, relative to point $\mathrm{P}$ (where $\alpha, \beta$ and $z$ are arbitrary), as a function of $q_{x}, q_{y}, q_{p}, \alpha, \beta$ and $z$.

The matrix notation of such a transformation is $Q=\boldsymbol{O}_{y}(\beta)$. $\boldsymbol{O}_{x}(\alpha) \cdot\left(\boldsymbol{q}+\boldsymbol{z}_{3}\right)$ that can be itemized as

$\left(\begin{array}{l}Q_{x} \\ Q_{y} \\ Q_{z}\end{array}\right)=\left(\begin{array}{ccc}\cos \beta & 0 & \sin \beta \\ 0 & 1 & 0 \\ -\sin \beta & 0 & \cos \beta\end{array}\right) \cdot\left(\begin{array}{ccc}1 & 0 & 0 \\ 0 & \cos \alpha & \sin \alpha \\ 0 & -\sin \alpha & \cos \alpha\end{array}\right) \cdot\left(\begin{array}{c}q_{x} \\ q_{y} \\ q_{z}+z\end{array}\right) \cdot(1)$

Movement of any reference point $Q_{\mathrm{q}}$ with constant coordinates $q\left[q_{x}, q_{y}, q_{2}\right]$ to a new point $Q=\left[Q_{x}, Q_{y}, Q_{2}\right]$ is represented by a change of the parameters $\alpha, \beta$ and \%. The required changes in the lengths of the individual telescopic rods, necessary for the implementation of such movement, are derived in [7].

Let $A_{0}$ be the change in length of rod AA', the change in length of rod BB' be $A_{1}$ and the change in length of rod CC' be $A_{-1}$ (see Figure 2):

$$
\begin{aligned}
& A_{-1}=\sqrt{R^{2}+r^{2}+z^{2}-2 R\left(\frac{1}{4} r \cdot \cos \beta+\cos \alpha \cdot\left(\frac{3}{4} r-\frac{1}{2} z \cdot \sin \beta\right)-\frac{\sqrt{3}}{2} \sin \alpha \cdot\left(z+\frac{1}{2} r \cdot \sin \beta\right)\right)}(2) \\
& A_{0}=\sqrt{\mathrm{R}^{2}+r^{2}+z^{2}-2 R(r \cdot \cos \beta+z \cdot \sin \beta \cdot \cos \alpha)} \\
& A_{1}=\sqrt{R^{2}+r^{2}+z^{2}-2 R\left(\frac{1}{4} r \cdot \cos \beta+\cos \alpha \cdot\left(\frac{3}{4} r-\frac{1}{2} z \cdot \sin \beta\right)+\frac{\sqrt{3}}{2} \sin \alpha \cdot\left(z+\frac{1}{2} r \cdot \sin \beta\right)\right)}(4)
\end{aligned}
$$

where free parameters $\alpha, \beta$ and $z$ must meet the following conditions:

$$
z=-q_{z}+S \sqrt{Q_{x}^{2}+Q_{y}^{2}+Q_{z}^{2}-q_{x}^{2}-q_{y}^{2}} ;
$$

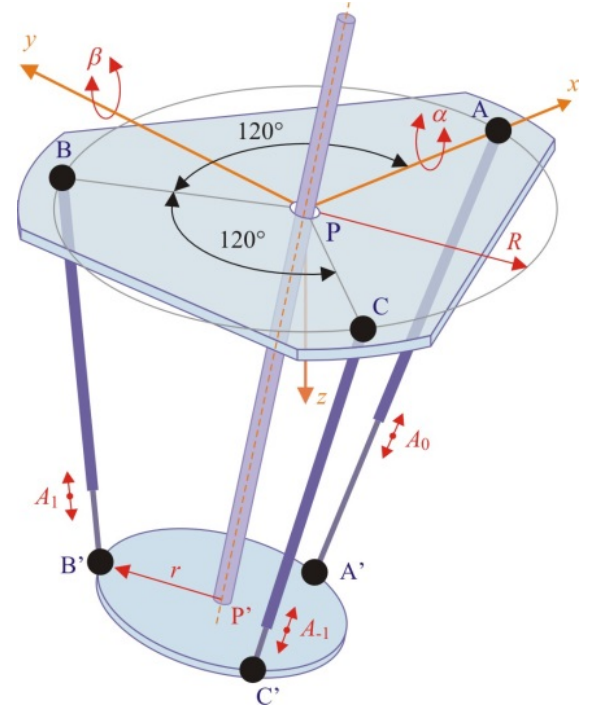

Figure 2. Schematic representation of telescopic rods, joints and platforms.

$$
\begin{aligned}
& \sin \beta=\frac{-q_{x} \cdot Q_{z}+K \sqrt{Q_{x}^{2}+Q_{z}^{2}-q_{x}^{2}} \cdot Q_{x}}{Q_{x}^{2}+Q_{z}^{2}} ; \\
& \cos \beta=\frac{q_{x} \cdot Q_{x}+K \sqrt{Q_{x}^{2}+Q_{z}^{2}-q_{x}^{2}} \cdot Q_{z}}{Q_{x}^{2}+Q_{z}^{2}} ; \\
& \sin \alpha=\frac{Q_{y} \cdot S \cdot \sqrt{Q_{x}^{2}+Q_{y}^{2}+Q_{z}^{2}-q_{x}^{2}-q_{y}^{2}}-K \cdot q_{y} \cdot \sqrt{Q_{x}^{2}+Q_{z}^{2}-q_{x}^{2}}}{Q_{x}^{2}+Q_{y}^{2}+Q_{z}^{2}-q_{x}^{2}} ; \\
& \cos \alpha=\frac{K \cdot S \sqrt{Q_{x}^{2}+Q_{y}^{2}+Q_{z}^{2}-q_{y}^{2}-q_{x}^{2}} \cdot \sqrt{Q_{x}^{2}+Q_{z}^{2}-q_{x}^{2}}+Q_{y} \cdot q_{y}}{Q_{x}^{2}+Q_{y}^{2}+Q_{z}^{2}-q_{x}^{2}} ;
\end{aligned}
$$

constants $K$ and $S$ are equal to 1 in this case.

\section{INFLUENCES THAT AFFECT THE REACHING OF THE DESIRED POSITION}

The positioning accuracy of any manufacturing device represents the closeness between the actual reached position of the end effector and a programmed position, specified by the control system. For PKS, the effector's endpoint is the point at the end of the central rod, respectively it is the precisely defined point on the tool or technology head [5], [6]. In our case, the point $P^{\prime}$ is considered.

Based on a theoretical analysis, one can summarize three types of errors affecting the reaching of the desired position by the PKS effector. The geometrical errors arise due to inaccuracies in manufacturing, inaccurate relative position of individual elements or due to wear of the joints. The stiffness errors originate from elasticity of joints between individual elements as well as from flexures caused by the own weights of individual elements or by an external load. Their magnitudes depend on the actual position of the effector. The thermal errors arise from thermal stress and dilatation of elements due to heat generated by internal or external sources, e.g. motors, bearings, etc. [11]-[18].

To document the complexity of the uncertainty calculation of reaching the desired position of the point $Q$, let us summarize the list of geometrical parameters that contribute to the overall uncertainty of reaching the desired position: 
a) distance of joints from the centre of the fixed platform, i.e. the distances AP, BP, CP (position of joints approximated by a circle with radius $R$ );

b) relative positions of joints at the fixed platform, i.e. the distance of points $\mathrm{CA}, \mathrm{CB}, \mathrm{BA}$;

c) distance of joints against the centre of the movable platform, i.e. the distances A'P', B'P', C'P' (position of joints approximated by a circle with radius $r$ );

d) distance of joints at the movable platform, ie. the distances C'A', C'B', B'A';

e) distance of the fixed platform and the movable one at the central rod, i.e. the distance PP';

f) if the effector is mounted, the distance between the effector's endpoint and the point of effector fixation at the movable platform P' (it is actually a determination of the vector $\left.q_{[}\left[q_{x}, q_{p}, q_{x}\right]\right)$;

g) lengths of individual telescopic rods, i.e. the distances AA', BB', CC'.

\section{METHODOLOGY FOR DETERMINATION OF THE DESIRED POSITION}

If the device designer knows the theoretically achievable positioning accuracy, he has an important opportunity to influence critical pieces of equipment in the process of the construction work. An uncertainty balance will help to identify the most significant influences on the theoretically achievable positioning accuracy of the effector, which opens up the possibility of corrective interventions into the structure. Only geometrical influences on the overall uncertainty are considered in the first phase, as shown in the further text. For the sake of simplicity we do not consider the covariances among the individual parameters.

Let a little change of the endpoint position be given as the product of the Jacobian of the tangential displacement in the direction of motion and a dimensionless vector of rotations and displacement. This is described by the following equation:

$$
\left(\begin{array}{l}
d Q_{x} \\
d Q_{y} \\
d Q_{z}
\end{array}\right)=\left(\begin{array}{ccc}
\frac{\partial Q_{x}}{\partial \alpha} & \frac{\partial Q_{x}}{\partial \beta} & \frac{\partial Q_{x}}{\partial z} \\
\frac{\partial Q_{y}}{\partial \alpha} & \frac{\partial Q_{y}}{\partial \beta} & \frac{\partial Q_{y}}{\partial z} \\
\frac{\partial Q_{z}}{\partial \alpha} & \frac{\partial Q_{z}}{\partial \beta} & \frac{\partial Q_{z}}{\partial z}
\end{array}\right) \cdot\left(\begin{array}{c}
d \alpha \\
d \beta \\
d z
\end{array}\right)
$$

The matrix in (5) is denoted as $\boldsymbol{M}_{3 \times 3}$. We obtain its elements later by partial derivation of (1).

A marginal change of the vector of rotations and translation from (4) depends on a limit change of lengths of the telescopic rods and radii $r$ and $R$, as given by the following equation:

$$
\left(\begin{array}{c}
d \alpha \\
d \beta \\
d z
\end{array}\right)=\left(\begin{array}{ccccc}
\frac{\partial \alpha}{\partial A_{0}} & \frac{\partial \alpha}{\partial A_{1}} & \frac{\partial \alpha}{\partial A_{-1}} & \frac{\partial \alpha}{\partial r} & \frac{\partial \alpha}{\partial \mathrm{R}} \\
\frac{\partial \beta}{\partial A_{0}} & \frac{\partial \beta}{\partial A_{1}} & \frac{\partial \beta}{\partial A_{-1}} & \frac{\partial \beta}{\partial r} & \frac{\partial \beta}{\partial \mathrm{R}} \\
\frac{\partial z}{\partial A_{0}} & \frac{\partial z}{\partial A_{1}} & \frac{\partial z}{\partial A_{-1}} & \frac{\partial z}{\partial r} & \frac{\partial z}{\partial \mathrm{R}}
\end{array}\right) \cdot\left(\begin{array}{c}
d A_{0} \\
d A_{1} \\
d A_{-1} \\
d r \\
d \mathrm{R}
\end{array}\right)
$$

The matrix in (6) is designated as $\boldsymbol{M}_{3 \times 5}$. The telescopic rod lengths from (2) to (4) will be used for the calculation of its elements. Their shapes are rather complicated for partial derivation, so that they will be adapted. Since the lengths of the rods cannot be negative, we can square them and find their appropriate linear combinations to get the simplest relations equivalent to (2) to (4). Three equations can be obtained in this way

$$
\begin{aligned}
& \frac{-A_{0}^{2}-A_{1}^{2}-A_{-1}^{2}}{3}+r^{2}+\mathrm{R}^{2}+z^{2}-r \cdot \mathrm{R} \cdot \cos \alpha-r \cdot \mathrm{R} \cdot \cos \beta=0 \\
& -\frac{A_{-1}^{2}-A_{1}^{2}}{\sqrt{3}}+2 \mathrm{R} \cdot \mathrm{z} \cdot \sin \alpha+r \cdot \mathrm{R} \cdot \sin \alpha \cdot \sin \beta=0, \\
& \frac{2 A_{0}^{2}-A_{1}^{2}-A_{-1}^{2}}{3}+2 \mathrm{R} \cdot \mathrm{z} \cdot \cos \alpha \cdot \sin \beta+r \cdot \mathrm{R} \cdot \cos \beta-r \cdot \mathrm{R} \cdot \cos \alpha=0 .
\end{aligned}
$$

Let us denote the left sides of (7) to (9) as functions $L_{1}, L_{2}$, $L_{3}$ that depend on parameters $A_{0}, A_{1}, A_{-1}, \alpha, \beta, r, r, \mathrm{R}$. We will consider the movement of point $\mathrm{Q}$ in time $t$ that will be marginally close to 0 and parameters $A_{0}, A_{1}, A_{-1}, \alpha, \beta, z, r, \mathrm{R}$ will depend on time $t$ as well.

If partial derivation of the left sides of (7) to (9) is carried out, similar to obtaining derivatives of the implicit function (parameters $\alpha, \beta$, z are derived from parameters $A_{0}, A_{1}, A_{-1}, r$, $R)$, the following equation is found:

$\boldsymbol{W}_{3 \times 3} \cdot \boldsymbol{M}_{3 \times 5} \cdot \boldsymbol{W}_{5 \times 1}+\boldsymbol{W}_{3 \times 5} \cdot \boldsymbol{W}_{5 \times 1}=\mathbf{0}$

where

$\boldsymbol{W}_{3 \times 3}=\left(\begin{array}{ccc}\frac{\partial L_{1}}{\partial \alpha} & \frac{\partial L_{1}}{\partial \beta} & \frac{\partial L_{1}}{\partial z} \\ \frac{\partial L_{2}}{\partial \alpha} & \frac{\partial L_{2}}{\partial \beta} & \frac{\partial L_{2}}{\partial z} \\ \frac{\partial L_{3}}{\partial \alpha} & \frac{\partial L_{3}}{\partial \beta} & \frac{\partial L_{3}}{\partial z}\end{array}\right)$

$=\left(\begin{array}{ccc}r \mathrm{R} \sin \alpha & r \mathrm{R} \sin \beta & 2 z \\ \mathrm{R} \cos \alpha(2 z+r \sin \beta) & r \mathrm{R} \sin \alpha \cdot \cos \beta & 2 \mathrm{R} \sin \alpha \\ \mathrm{R} \sin \alpha(r-2 z \sin \beta) & \mathrm{R}(2 z \cos \alpha \cos \beta-r \sin \beta) & 2 \mathrm{R} \cos \alpha \sin \beta\end{array}\right)$

$W_{3 \times 5}=\left(\begin{array}{lllll}\frac{\partial L_{1}}{\partial A_{0}} & \frac{\partial L_{1}}{\partial A_{1}} & \frac{\partial L_{1}}{\partial A_{-1}} & \frac{\partial L_{1}}{\partial r} & \frac{\partial L_{1}}{\partial R} \\ \frac{\partial L_{2}}{\partial A_{0}} & \frac{\partial L_{2}}{\partial A_{1}} & \frac{\partial L_{2}}{\partial A_{-1}} & \frac{\partial L_{2}}{\partial r} & \frac{\partial L_{2}}{\partial R} \\ \frac{\partial L_{3}}{\partial A_{0}} & \frac{\partial L_{3}}{\partial A_{1}} & \frac{\partial L_{3}}{\partial A_{-1}} & \frac{\partial L_{3}}{\partial r} & \frac{\partial L_{3}}{\partial R}\end{array}\right)$

$=\left(\begin{array}{ccccc}\frac{-2 A_{0}}{3} & \frac{-2 A_{1}}{3} & \frac{-2 A_{-1}}{3} & 2 r-R(\cos \alpha+\cos \beta) & 2 R-r(\cos \alpha+\cos \beta) \\ 0 & \frac{2 A_{1}}{\sqrt{3}} & \frac{-2 A_{-1}}{\sqrt{3}} & R \sin \alpha \sin \beta & \sin \alpha(2 z+r \cdot \sin \beta) \\ \frac{4 A_{0}}{3} & \frac{-2 A_{1}}{3} & \frac{-2 A_{-1}}{3} & R(-\cos \alpha+\cos \beta) & r \cos \beta+\cos \alpha(-r+2 z \sin \beta)\end{array}\right)$

$\boldsymbol{W}_{5 \times 1}=\left(\begin{array}{c}\dot{A}_{0}(0) \\ \dot{A}_{1}(0) \\ \dot{A}_{-1}(0) \\ \dot{r}(0) \\ \dot{R}(0)\end{array}\right)$.

The relation (10) can be transformed to

$\left(\boldsymbol{W}_{3 \times 3} \cdot \boldsymbol{M}_{3 \times 5}+\boldsymbol{W}_{3 \times 5}\right) \cdot \boldsymbol{W}_{5 \times 1}=\boldsymbol{O}$

Matrix $\boldsymbol{M}_{3 \times 5}$ is calculated from (11):

$\boldsymbol{M}_{3 \times 5}=-\boldsymbol{W}_{3 \times 3}^{-1} \cdot \boldsymbol{W}_{3 \times 5}$

Let us return to the expression of matrix $\boldsymbol{M}_{3 \times 3}$ from (5). When multiplying (1) from left by matrix $\boldsymbol{O}_{\gamma}^{T}(\beta)$, we get: 


$$
O_{y}^{T}(\beta) \cdot Q=O_{y}^{T}(\beta) \cdot O_{y}(\beta) \cdot O_{x}(\alpha) \cdot\left(q+z e_{3}\right)
$$

and subsequently

$-O_{y}^{T}(\beta) \cdot Q+O_{x}(\alpha) \cdot\left(q+z e_{3}\right)=0$

After multiplying of (3) we get

$-q_{x}+Q_{x} \cos \beta-Q_{z} \sin \beta=0$

$-Q_{y}+q_{y} \cdot \cos \alpha+\left(q_{z}+z\right) \sin \alpha=0$

$-\left(q_{z}+z\right) \cdot \cos \alpha+Q_{z} \cdot \cos \beta+q_{y} \cdot \sin \alpha+Q_{x} \cdot \sin \beta=0$.

If we denote the left sides of (15) to (17) as $H_{1}, H_{2}, H_{3}$ and we carry out their partial derivatives, we get the following matrices:

$$
\boldsymbol{F}_{3 \times 3}=\left(\begin{array}{lll}
\frac{\partial H_{1}}{\partial Q_{x}} & \frac{\partial H_{1}}{\partial Q_{y}} & \frac{\partial H_{1}}{\partial Q_{z}} \\
\frac{\partial H_{2}}{\partial Q_{x}} & \frac{\partial H_{2}}{\partial Q_{y}} & \frac{\partial H_{2}}{\partial Q_{z}} \\
\frac{\partial H_{3}}{\partial Q_{x}} & \frac{\partial H_{3}}{\partial Q_{y}} & \frac{\partial H_{3}}{\partial Q_{z}}
\end{array}\right)=\left(\begin{array}{ccc}
\cos \beta & 0 & -\sin \beta \\
0 & -1 & 0 \\
\sin \beta & 0 & \cos \beta
\end{array}\right)
$$

and

$$
\begin{gathered}
\boldsymbol{G}_{3 \times 3}=\left(\begin{array}{ccc}
\frac{\partial H_{1}}{\partial \alpha} & \frac{\partial H_{1}}{\partial \beta} & \frac{\partial H_{1}}{\partial z} \\
\frac{\partial H_{2}}{\partial \alpha} & \frac{\partial H_{2}}{\partial \beta} & \frac{\partial H_{2}}{\partial z} \\
\frac{\partial H_{3}}{\partial \alpha} & \frac{\partial H_{3}}{\partial \beta} & \frac{\partial H_{3}}{\partial z}
\end{array}\right) \\
=\left(\begin{array}{ccc}
\left(q_{z}+z\right) \cos \alpha-q_{y} \sin \alpha & -Q_{x} \sin \beta-Q_{z} \cos \beta & 0 \\
q_{y} \cos \alpha+\left(q_{z}+z\right) \sin \alpha & Q_{x} \cos \beta-Q_{z} \sin \beta & -\cos \alpha
\end{array}\right)
\end{gathered}
$$

Analogically to $\boldsymbol{M}_{3 \times 5}$, we can calculate the matrix $\boldsymbol{M}_{3 \times 3}$ [8] as the product of matrices $-\boldsymbol{F}_{3 \times 3}^{-1}$ and $\boldsymbol{G}_{3 \times 3}$. Subsequently we get the matrix of sensitivity coefficients as the product of matrices $\boldsymbol{M}_{3 \times 3}$ and $\boldsymbol{M}_{3 \times 5}$ :

$$
\boldsymbol{A}_{3 \times 5}=\boldsymbol{F}_{3 \times 3}^{-1} \cdot\left(-\boldsymbol{G}_{3 \times 3}\right) \cdot \boldsymbol{W}_{3 \times 3}^{-1} \cdot\left(-\boldsymbol{W}_{3 \times 5}\right)=\boldsymbol{M}_{3 \times 3} \cdot \boldsymbol{M}_{3 \times 5} .
$$

Matrix $\boldsymbol{A}_{3 \times 5}$ in (20) is used for the calculation of estimates of uncertainties of indirectly measured parameters. The covariance matrix of those estimates is

$\boldsymbol{U}_{y}=\boldsymbol{A} \cdot \boldsymbol{U}_{x} \cdot \boldsymbol{A}^{\mathrm{T}}$,

where matrix $\boldsymbol{U}_{x}$ in the form

$$
\boldsymbol{U}_{x}=\left(\begin{array}{lllll}
u_{x_{1}, x_{1}}^{2} & u_{x_{1}, x_{2}} & u_{x_{1}, x_{3}} & u_{x_{1}, x_{4}} & u_{x_{1}, x_{5}} \\
u_{x_{2}, x_{1}} & u_{x_{2}, x_{2}}^{2} & u_{x_{2}, x_{3}} & u_{x_{2}, x_{4}} & u_{x_{2}, x_{5}} \\
u_{x_{3}, x_{1}} & u_{x_{3}, x_{2}} & u_{x_{3}, x_{3}}^{2} & u_{x_{3}, x_{4}} & u_{x_{3}, x_{5}} \\
u_{x_{4}, x_{1}} & u_{x_{4}, x_{2}} & u_{x_{4}, x_{3}} & u_{x_{4}, x_{4}}^{2} & u_{x_{4}, x_{5}} \\
u_{x_{5}, x_{1}} & u_{x_{5}, x_{2}} & u_{x_{5}, x_{3}} & u_{x_{5}, x_{4}} & u_{x_{5}, x_{5}}^{2}
\end{array}\right)
$$

is a known covariance matrix of the random vector $\boldsymbol{x}=\left(x_{1}, x_{2}\right.$, $\left.x_{3}, x_{4}, x_{5}\right)=\left(A_{0}, A_{1}, A_{-1}, r, R\right)$, where $u_{x_{i}}$ is the standard uncertainty of the estimate $x_{i}$ of quantity $X_{i}, i=1,2, \ldots 5$, $u_{x_{i, j}}$ is the covariance between estimates $x_{i}$ and $x_{j}, i=1,2, . ., 5$, $j=1,2, . ., 5$.

The position uncertainty of any point $Q$ in the workspace can be calculated, if the matrix $\boldsymbol{U}_{x}$ is known.

Let us briefly introduce the principle how to estimate the matrix $\boldsymbol{U}_{y}$. The matrix $\boldsymbol{A}_{3 \times 5}\left(Q_{x}, Q_{y}, Q_{*}\right)$ represents a functional relationship of position of the reference point $Q$, i.e. the position of the Tricept. As we know the numerical values of $Q_{x}$ $Q_{3}, Q_{0}$, we can quantify the matrix of the partial derivatives of $\boldsymbol{A}_{3 \times 5}$.

Let $\boldsymbol{U}_{x}$ be a known constant symmetric matrix of $5 \times 5$ type, and $\boldsymbol{U}_{y}$ be an unknown symmetric matrix of $3 \times 3$ type that we want to determine and is given by (21). It is clear that the matrix $\boldsymbol{U}_{y}$ is correlated with the position of the point $Q\left[Q_{x}, Q_{y}\right.$, $Q_{2}$. We want to find the intervals for values of the matrix $\boldsymbol{U}_{y}$, when considering that $Q_{x}, Q_{y}, Q_{z}$ may take any value, depending on how the reference point $Q$ moves in some regular subspace (let it be a cube for purposes of this estimate, see Figure 5) of the overall workspace.

To analyze the impact of individual components of the known matrix $\boldsymbol{U}_{x}$ on elements of the matrix $\boldsymbol{U}_{y}$, we employ the linearity of this dependence (for fixed $Q$ ). We will just consider only the base symmetrical matrices $\boldsymbol{U}_{x}$ such that there is always only one element equal to 1 (for diagonal elements; outside the diagonal we consider also symmetrically associated elements). This element or these elements are multiplied by the respective weighting coefficient for the particular matrix $\boldsymbol{U}_{x}$. If we fix the angles $\alpha$ and $\beta$, a virtual beam arises in the cube, along which the reference point will move.

When the reference point $Q$ moves along the beam, we want numerically determine the intervals for the values of elements of the matrix $\boldsymbol{U}_{y}$. Each element value of the matrix $\boldsymbol{U}_{y}$ (since it is a symmetrical matrix, 6 different elements are considered) can be precisely expressed using a formula that is represented by the sum of 6 square roots of polynomials of the variable $z$ divided by other polynomials also dependent on $₹$ (their shape is too extensive for the length of this paper). The derivative of this formula to $z$ can be algebraically adjusted (it shall be multiplied by analogous expressions, where we change only marks of generated roots, thus, removing roots and a multiplied derivative, obtained in this way, simplifies the polynomial). It is true that any stationary point of the original expression is also the root of this polynomial at the same time. It is sufficient to evaluate the expression for a particular beam only in the roots of this polynomial (if they overlap the workspace) and also in the endpoints of the beam, defined by the workspace borders. Among them we find the minimum and maximum, which will form the search interval for the selected element of matrix $\boldsymbol{U}_{y}$, for fixed angles $\alpha$ and $\beta$ and a base matrix $\boldsymbol{U}_{x}$.

Resulting intervals that represent the impact of elements of the matrix $\boldsymbol{U}_{x}$ in the overall workspace can be obtained by uniting intervals for all permissible values of $\alpha$ and $\beta$. To do so, a sufficiently fine division of the workspace, with a properly chosen step only in a two-dimensional area that is created by projection of the workspace on $₹$ (along rays) into the variables $\alpha$ and $\beta$, must be considered. The impact of each element of the matrix $\boldsymbol{U}_{x}$ can be displayed using a three-dimensional function (see Figures 3 to 10). A search estimate of the matrix $\boldsymbol{U}_{y}$ is obtained as a matrix of ordered pairs (minimum and maximum impacts of components of the matrix $\boldsymbol{U}_{x}$ ). 


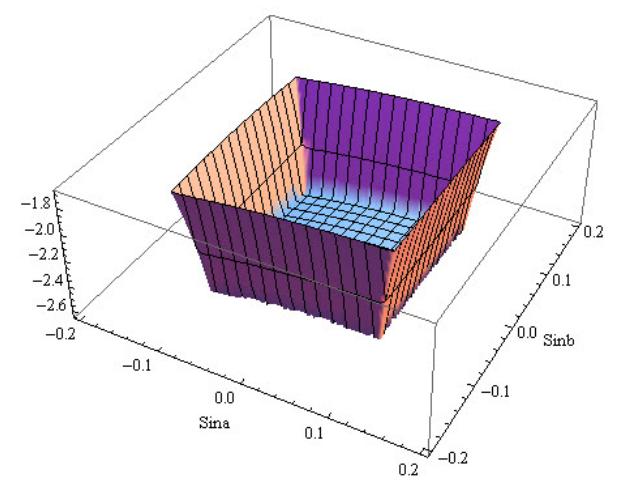

Figure 3. Influence of element $\boldsymbol{U}_{x}[1,2]$ on minimum value of the matrix $\boldsymbol{U}_{y}[1,1]$.

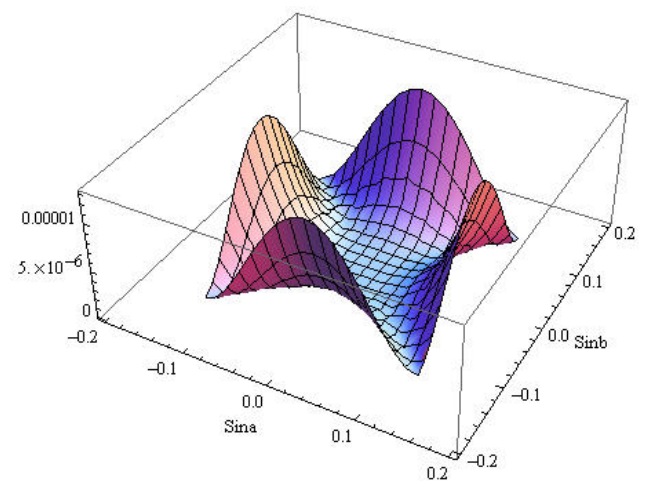

Figure 5. Influence of element $\boldsymbol{U}_{x}[4,4]$ on maximum value of the matrix $\boldsymbol{U}_{y}[1,1]$.

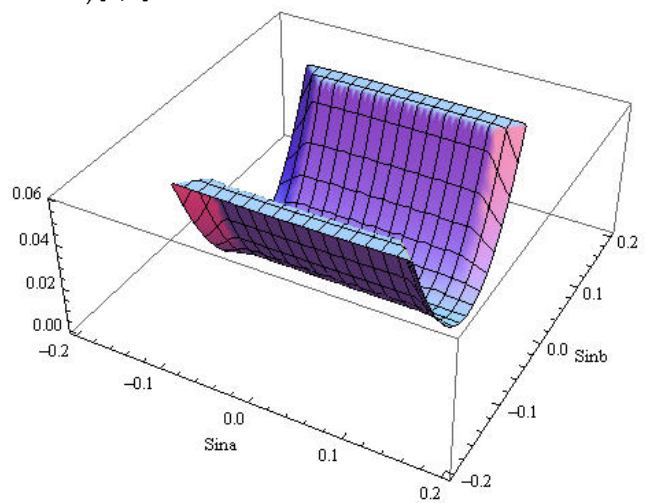

Figure 7. Influence of element $\boldsymbol{U}_{x}[5,5]$ on maximum value of the matrix $\boldsymbol{U}_{y}[1,1]$.

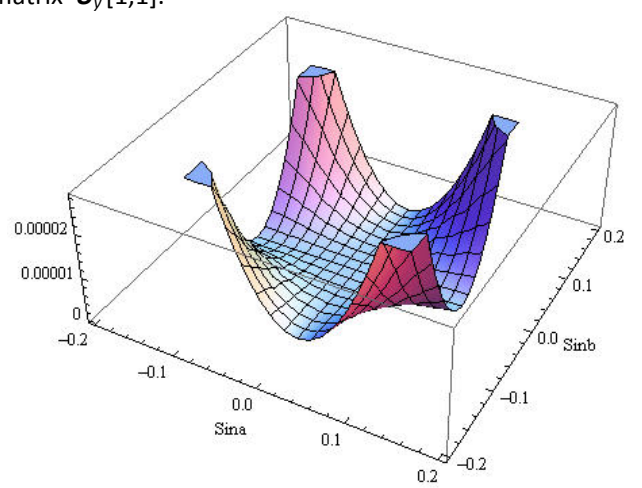

Figure 9. Influence of element $\boldsymbol{U}_{x}[4,4]$ on minimum value of the matrix $\boldsymbol{U}_{y}[2,2]$.

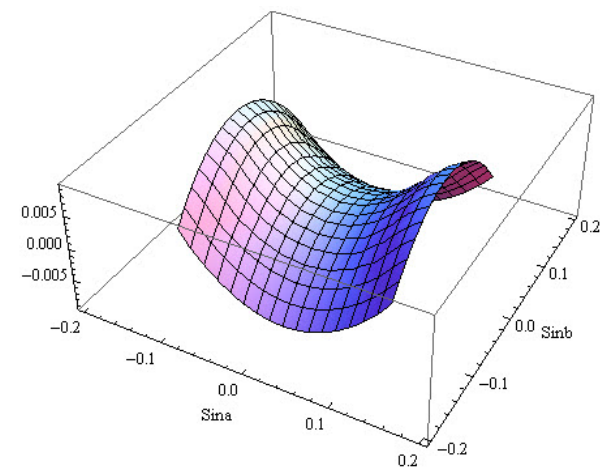

Figure 4. Influence of element $\boldsymbol{U}_{x}[1,4]$ on maximum value of the matrix $\boldsymbol{U}_{y}[1,1]$.

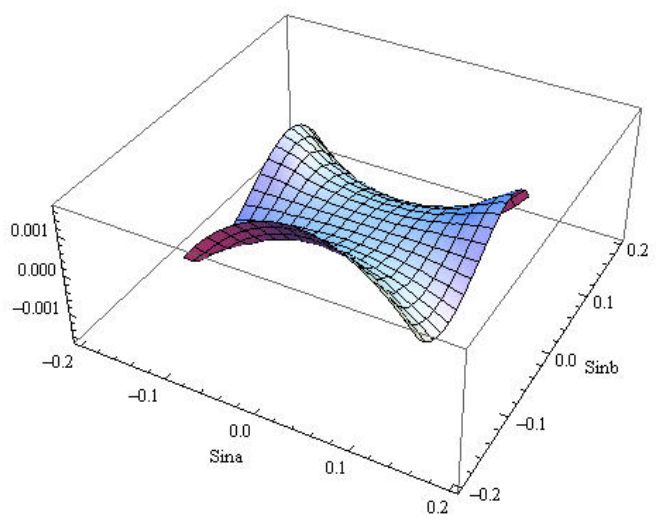

Figure 6 . Influence of element $\boldsymbol{U}_{x}[4,5]$ on minimum value of the matrix $\boldsymbol{U}_{y}[1,1]$

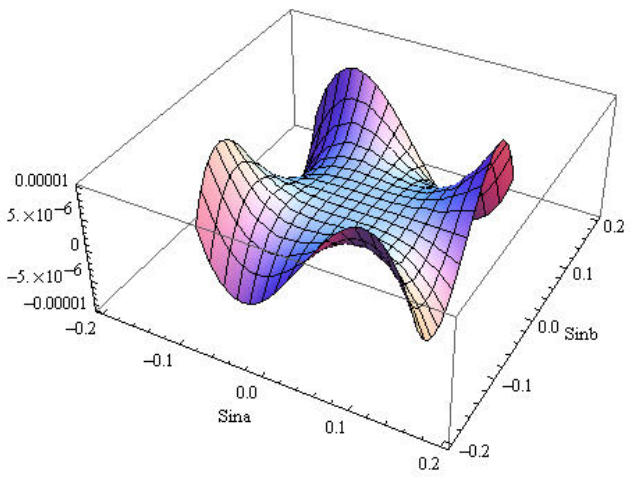

Figure 8. Influence of element $\boldsymbol{U}_{x}[4,4]$ on minimum value of the matrix $\boldsymbol{U}_{y}[1,2]$.

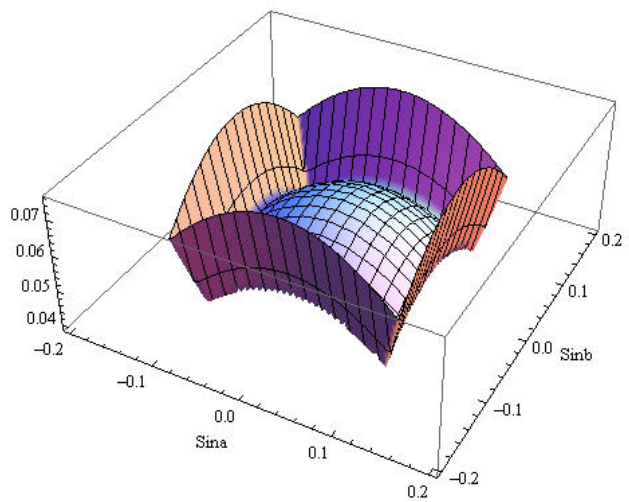

Figure 10. Influence of element $\boldsymbol{U}_{x}[5,5]$ on minimum value of the matrix $\boldsymbol{U}_{y}[3,3]$. 
They represent the range of values of elements of the matrix $\boldsymbol{U}_{y}$. Estimates of 15 base matrices $\boldsymbol{U}_{x}$ can be linearly combined and can thus provide an estimate for any general (non-base) symmetric matrix $\boldsymbol{U}_{x}$.

Using the software system MATHEMATICA, we created a program to search the entire workspace (or its subset thereof) and to estimate the matrix $\boldsymbol{U}_{y}$. To do so, the matrix $\boldsymbol{U}_{x}$ must be specified and the required division of the workspace must be selected.

For example for

$$
\mathbf{U}_{x}=\left(\begin{array}{ccccc}
(0.005 / \sqrt{3})^{2} & 0 & 0 & 0 & 0 \\
0 & (0.005 / \sqrt{3})^{2} & 0 & 0 & 0 \\
0 & 0 & (0.005 / \sqrt{3})^{2} & 0 & 0 \\
0 & 0 & 0 & (0.01 / \sqrt{3})^{2} & 0 \\
0 & 0 & 0 & 0 & (0.01 / \sqrt{3})^{2}
\end{array}\right)
$$

as well as for the reference point $Q_{q}$, identical to point P' (when $\left.\boldsymbol{q}=\left[q_{x}, q_{y}, q_{z}\right]=[0,0,0]\right)$, we found the estimate of $\boldsymbol{U}_{y}$ valid for all possible positions of the point $Q$ over the regular space (a cube) that fully fits into the workspace:

$$
\begin{aligned}
& \left(\begin{array}{ccc}
0.0000185 & -7.0896 \times 10^{-6} & -7.3262 \times 10^{-6} \\
-7.0896 \times 10^{-6} & 0.0000185 & -7.3394 \times 10^{-6} \\
-7.3262 \times 10^{-6} & -7.3394 \times 10^{-6} & 0.0000038
\end{array}\right) \\
& \leq \boldsymbol{U}_{y} \leq\left(\begin{array}{ccc}
0.0000368 & 7.0896 \times 10^{-6} & 7.2188 \times 10^{-6} \\
7.0896 \times 10^{-6} & 0.0000370 & 7.3394 \times 10^{-6} \\
7.2188 \times 10^{-6} & 7.3394 \times 10^{-6} & 0.0000138
\end{array}\right)
\end{aligned}
$$

The diagonal of the covariance matrix $\boldsymbol{U}_{y}$ contains estimates of uncertainties squares $u_{Q_{x}}^{2}, u_{Q_{y}}^{2} u_{Q_{z}}^{2}$, valid over the whole considered cube (see Figure 11 and Table 1).

\section{CONCLUSION}

This paper analyzed various issues related to the control of
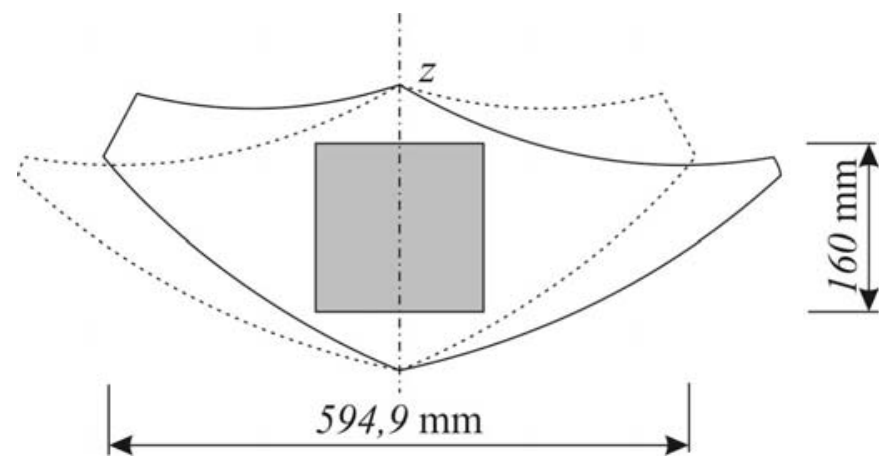

Figure 11. Scheme of the cube that represents the biggest regular object in the workspace.

Table 1. Considered parameters and their uncertainties.

\begin{tabular}{c|c|c|c|c}
\hline $\begin{array}{c}\text { Para- } \\
\text { meter }\end{array}$ & Value / mm & $\begin{array}{c}\text { Permissible } \\
\text { deviation / mm }\end{array}$ & $\begin{array}{c}\text { Probability } \\
\text { distribution }\end{array}$ & $\begin{array}{c}\text { Uncertainty / } \\
\mathbf{m m}\end{array}$ \\
\hline$A_{0}$ & 568 to 858 & 0.005 & Rectangular & 0.0029 \\
\hline$A_{1}$ & 568 to 858 & 0.005 & Rectangular & 0.0029 \\
\hline$A_{-1}$ & 568 to 858 & 0.005 & Rectangular & 0.0029 \\
\hline$R$ & 330 & 0.010 & Rectangular & 0.0058 \\
\hline$r$ & 140 & 0.010 & Rectangular & 0.0058 \\
\hline
\end{tabular}

structures with parallel kinematics, especially that relating to the positioning accuracy. The function describing the lengthening and shortening of the individual telescopic drives and the desired setpoint is non-linear. Because of this, the equations cannot be partially derived, making the uncertainty analysis unfeasible. In order to overcome this difficulty, the employment of an approach using infinite geometrical changes in the parameters is suggested. The marginal values for uncertainties were calculated here, suggesting that the achievable positioning accuracy is not constant for all setpoints within the workspace of the Tricept device.

Further research will be focused on finishing the Tricept prototype, in which the presented analysis will provide orientation for the designers to assess end effector accuracy. The investigation of the contributing sources of uncertainties and a practical verification of the results of this article remains a further challenge.

\section{ACKNOWLEDGEMENT}

This paper was created within the research grant supported by the Scientific grant agency of the Ministry for Education, grant number VEGA 1/0604/15.

\section{REFERENCES}

[1] PKM Tricept, http://www.pkmtricept.com.

[2] J.L. Olazagoitia, S. Wyatt, "New PKM Tricept T9000 and its application to flexible manufacturing at aerospace industry", in: SAE Technical Paper, 2007.

[3] G. Pritschow, "Parallel kinematic machines (PKM) - limitations and new solutions", Annals of the CIRP, 2000, 49 (1), pp. 275280.

[4] J.P. Merlet, Parallel Robots, Springer Series: Solid Mechanics and Its Applications, 2006, ISBN 978-94-010-9587-7

[5] I. Onderová, L. Kolláth, "Testing and verification of selected technological parameters of the PKS", Proc. of $15^{\text {th }}$ International Carpathian Control Conference - IEEE, May 2830, 2014, Velké Karlovice, Czech republic, pp. 398-402.

[6] L. Kolláth, M. Halaj, E. Kureková, "Positioning accuracy of nonconventional production machines", Proc. of 19th IMEKO World Congress, Sept. 6-11, 2009, Lisbon, Portugal, pp. 20992102.

[7] M. Omachelová, I. Martišovitš, E. Kureková, L. Kolláth, "Analytical expression of the lengths of tricept telescopic rods ejection", Proc. of 37th International Conference Instruments and Control, 2013, Ostrava, Czech republic, pp. 45-52.

[8] M. Omachelová, E. Kureková, M. Halaj, I. Martišovitš, "Theoretical aspects of control of the Tricept type parallel kinematic structure", Proc. of 15th International Carpathian Control Conference - IEEE, May 28-30, 2014, Velké Karlovice, Czech republic, pp. 393-397.

[9] S. Besnard, W. Khalil, "Identifiable Parameters for Parallel Robots Kinematic Calibration" IEEE International Conference on Robotics and Automation, 2001, pp. 2859-2865 vol (3).

[10] L. Kolláth, I. Martišovitš, M. Omachelová, "Solving the problems of workspace in parallel kinematic structure", Annals of the Faculty of Engineering Hunedoara, ISSN $1584-2673$, 2014, pp. 15-17.

[11] W. Knapp, "Measurement uncertainty and machine tool testing", CIRP Annals - Manufacturing Technology, 2002, 51 (1), pp. 459-462.

[12] H. Schwenke, et al., "Geometric error measurement and compensation of machines - An update", CIRP Annals Manufacturing Technology, 2008, 57 (2), pp. 660-675.

[13] S. Sartori, G.X. Zhang, „Geometric Error Measurement and Compensation of Machines". CIRP Annals - Manufacturing Technology, 19954, 4 (2), pp. 599-609. 
[14] A. Weckenmann, "The accuracy of coordinate measuring machines", Proc. of 9th IMEKO World Congress, 1982, May 24-28, West-Berlin, pp. 266-275.

[15] A. Balsamo, A. Meda, "Geometrical error compensation of coordinate measuring systems", Nanotechnology and Precision Engineering, 2006, ISSN 1672-6030, pp.83-91.

[16] M. Kušnerová, J. Valíček, M. Harničárová, T. Hryniewicz, K. Rokosz, Z. Palková, V. Václavík, M. Řepka, M. Bendová, “A Proposal for simplifying the method of evaluation of uncertainties in measurement result", Measurement Science Review, 2013, ISSN 1335 - 8871, 13 (1), pp. 1-6.
[17] K. Ostrowska, A. Gaska, J. Sladek, „Determining the uncertainty of measurement with the use of a Virtual Coordinate Measuring Arm" The International Journal of Advanced Manufacturing Technology, ISSN 1433-3015, 2014, 71 (1-4), pp.529-537.

[18] S. Aguado, J. Santolaria, D. Samper, J.J. “Aguilar, Influence of measurement noise and laser arrangement on measurement uncertainty of laser tracker multilateration in machine tool volumetric verification" Precision Engineering, Elsevier, ISSN 01416359, 2013, (4), pp. 929-943. 